\title{
The hydrodynamic forces on a circular cylinder in proximity to a wall with intermittent contact in steady current
}

\author{
Feifei Tong $^{\mathrm{a}}$, Liang Cheng ${ }^{\mathrm{a}, \mathrm{b},} \uparrow$, Hongwei $\mathrm{An}^{\mathrm{a}}$ and Terry Griffiths ${ }^{\mathrm{a}}$ \\ ${ }^{a}$ School of Civil, Environmental and Mining Engineering, The University of Western Australia, \\ 35 Stirling Highway, Perth, WA 6009, Australia \\ ${ }^{\mathrm{b}}$ DUT-UWA Joint Research Centre, State Key Laboratory of Coastal and Offshore Engineering, \\ Dalian University of Technology, \\ No. 2 Linggong Road, Dalian 116024, China
}

\begin{tabular}{l} 
ARTICLE INFO \\
Article history: \\
Received * \\
Accepted * \\
Editor-in-Chief: * \\
\hline Keywords: \\
hydrodynamic force \\
submarine pipeline \\
zebraic spanning \\
steady flow
\end{tabular}

ARTICLE INFO

\section{ABSTRACT}

We present a three-dimensional Large Eddy Simulations (LES) study on the hydrodynamics of a circular cylinder close to a wall. Intermittent gaps between the cylinder and wall, forming spanning and non-spanning sections along the span are considered. This geometry setting represents an idealized model of pipelines laid on an uneven seabed. As compared to that of the corresponding spanwise-uniform cylinders, the drag coefficient on spanning sections is generally smaller, while the drag on non-spanning sections is slightly larger. Large amplitudes of the sectional forces are observed, partially due to the variations of the gap flow under the spanned sections. Flow deflection from the non-spanning section to the spanning section induces changes in local force and pressure distributions. Despite these changes, the mean force on the cylinder with intermittent contact as a whole is shown to be reasonably estimated by pro rata of forces from spanwiseuniform cylinders over the parameter space considered in this study.

\section{Introduction}

The research on hydrodynamics around a horizontal circular cylinder near a plane boundary has been largely driven by pipeline engineering in the offshore oil and gas industry, which has been well documented in the monographs by Sumer \& Fredsøe (1997) and Zdravkovich (1997), as well as research papers by Sarpkaya (1976), Bearman \& Zdravkovich (1978), Zdravkovich (1985), Kozakiewicz, Fredsøe \& Sumer (1995), Lei, Cheng \& Kavanagh (1999) and Price et al. (2002), among others. Most of the studies considered a wall-cylinder configuration with planar twodimensional cross-sections, and the bed wall was either treated as smooth or smooth with grain roughness. While the flat-wall assumption is advantageous in separating the wall effects from the effects of diverse seafloor profiles (due to scour processes or the presence of rocky seafloor, for instance), the pipeline and seabed contacts are unlikely uniform. Field observations on subsea pipelines show variation in both as-laid embedment (e.g. Westgate \& White (2015)) and in the changing through-life contact due to self-burial processes after installation (e.g. Leckie et al. (2015)). However, the effect of these gap features on the hydrodynamics is not well-known, yet it is believed to be important especially when a small diameter pipe is considered, such as subsea cables.

In addition, many pipelines have to cross seafloor areas characterized by rocks, valleys and hills, in the presence of frequent severe storm events. This paper forms part of a wider research effort 
being undertaken by the University of Western Australia (UWA) using both physical and numerical testing of pipe behavior over artificially-created rocky seabed. The work includes lateral resistance of pipes on rocky seabed (Griffiths et al. 2017), the effect of seabed roughness on enhanced boundary layer thickness and the validity of existing hydrodynamic force models for small diameter cables (Cheng et al. 2016). In this paper, the effects of intermittent contact on hydrodynamic forces are investigated using a numerical approach.

The hydrodynamic characteristics on a circular cylinder subjected to steady current includes the drag coefficient $C_{D}$, lift coefficient $C_{L}$, and the oscillation frequency of the forces (such as due to vortex shedding), which are defined as,

$$
C_{D}=\frac{F_{x}}{0.5 \rho U_{\infty}^{2} D H}, C_{L}=\frac{F_{y}}{0.5 \rho U_{\infty}^{2} D H}, S t=\frac{f_{s} D}{U_{\infty}}
$$

where $F_{x}$ and $F_{y}$ are the forces in the in-line and cross-flow directions, respectively, $\rho$ the density of the fluid, $U_{\infty}$ the unaffected incoming fluid velocity outside the wall boundary layer, $D$ the diameter and $H$ is the length of the cylinder. The $f_{s}$ are normally referred to the vortex shedding frequency in the classic von Kármán vortex sheet, which can be measured by the dominant frequency in the lift force. However, for a pipe close to a wall as it will be seen later, it is noticed that the unsteadiness of the forces is not necessarily due to vortex shedding. The root-mean-square $(r m s)$ values of the $C_{D}$ and $C_{L}$, which describe the oscillation amplitude of the forces, are also important in characterizing the hydrodynamic features.

For a wall-free circular cylinder, the hydrodynamic forces and Strouhal number are mainly influenced by the Reynolds number $(R e)$,

$$
R e=\frac{U_{\infty} D}{v},
$$

where $v$ is the kinematic viscosity of the fluid. However, wall-proximity complicates the problem in the sense that it introduces a wall boundary layer. There is no surprise that the forces on a cylinder near a wall are also dependent on the distance $(e)$ between the wall and the cylinder, normally defined as gap ratio,

$$
G=\frac{e}{D}
$$

But for avoidance of confusion in this paper, $G$ is henceforth referred to as the cylinder elevation above the far-field seabed and it is independent of the configuration of local seabed contact under the cylinder.

From the observations by Leckie et al. (2015) it is recognized that a circular cylinder close to a plane boundary can experience global lowering relative to the far-field elevation of the plane bed, with local gaps under the cylinder occurring as deformations in the bed surface. These observations contrast with observations from other pipelines where embedment occurs due to sedimentation of the local seabed above the far-field bed elevation (Leckie et al. 2016) in the absence of significant pipeline lowering. In this work, the approach is adopted of treating the horizontal cylinder as variable in elevation above the bed, with and without contact generated as localized increases in bed elevation against the pipe as shown in Figure 1. 
For a circular cylinder above a plane wall, the general trend is that the $\bar{C}_{D}$ increases with increasing $G$, until it asymptotes to the value of an isolated cylinder at large $G(>2.0)$. Zdravkovich (1985) correlated the critical $G$ with the thickness of the boundary layer ( $\delta$, the distance from the wall to a point where the velocity reaches $99 \%$ of $U_{\infty}$ ). It was also found that $\bar{C}_{D}$ peaks at about $G=$ $0.5 \sim 0.6$ at about 1.2 times that on an isolated cylinder (Lei et al. 1999; Roshko, Steinolfson \& Chattoorgoon 1975; Zdravkovich 1985) and drops to less than $50 \%$ at zero $G$. The $\bar{C}_{L}$ is normally larger than 0 due to that the proximity of a wall moves the stagnation point on the cylinder downwards close to the wall. The variation of $\bar{C}_{L}$ with $G$ is characterized by two peaks. One is at close-to-zero $G$, when the suction pressure on the cylinder surface dominates; another one at around $G=0.1$, where the stagnation pressure dominates (Sumer \& Fredsøe 1997). However, for a turbulent boundary layer generated by trip-wires (Lei et al. 1999; Zdravkovich 1985), the cylinder experiences a negative lift for $G$ between $0.3 \sim 1.3$. For Strouhal number, $S t$, it increases with decreasing $G$, until the vortex shedding is fully suppressed. The reported critical $G$ for this change in vortex shedding behavior differs significantly among studies and falls into a wide range between $0.2 \sim 0.9$ (Buresti \& Lanciotti 1979; Taniguchi \& Miyakoshi 1990), depending on factors including $R e$.

For a partially buried cylinder, both hydrodynamic forces and the flow features have been reasonably researched (Jacobsen, Bryndum \& Bonde 1989; Cokgor \& Avci 2001; Gao et al. 2011; Akoz 2012). Due to its natural process, the hydrodynamic loading is usually studied concurrently with the bed-form evolution and the pipe-soil interaction ( $\mathrm{Xu}$ et al. 2010; Gao, Gu \& Jeng 2003). For a cylinder buried in an impermeable bed, the reduction in the force coefficient were found to be substantial, especially for the in-line force (Jacobsen et al. 1989). Probably as expected, the force coefficients decreased with increasing burial ratio for the steady current (Cokgor \& Avci 2001). As reported by Akoz (2012), rotational flows were observed close to the wall in the upstream of a partially buried circular cylinder and the starting point of flow separation in the upstream is strongly dependent on the burial ratio and $R e$. We note that no clear vortex shedding in the wake of the cylinder from the detailed PIV measurement (Akoz 2012), which is consistent with Bearman \& Zdravkovich (1978).

An, Cheng \& Zhao (2011) studied a partially buried cylinder in a permeable seabed subjected to combined oscillatory flow and steady current through two-dimensional (2D) numerical simulations. Different embedment depths besides the cylinder were considered for the first time. In agreement with Jacobsen et al. (1989), the in-line force reduces due to the reduction in exposed area normal to the flow. Interestingly, although seepage flow was found to be a few magnitudes smaller than the undisturbed inlet flow, it played a role in enlarging the in-line force.

Available research on hydrodynamics on a cylinder above an uneven wall is relatively rare. The unevenness of a seabed may lead to free spans of submarine pipelines, which are crucial for the structural behavior and safety (Ronold 1995), partially due to the change in hydrodynamic loading and the dynamic features of free spanning pipelines (Furnes \& Berntsen 2003). Jensen et al. (1990) experimentally investigated a pipeline placed on a frozen-scoured bed to study the hydrodynamics. The seabed profiles were taken from five characteristic stages of the scour process in currents. It was demonstrated that both $\bar{C}_{D}$ and $\bar{C}_{L}$ quickly reach the equilibrium values at an early stages of the scour process. The $\bar{C}_{D}$ was found to be about $20 \%$ smaller in the case of the scoured bed than that from plane wall boundary for $0.4 \leq G \leq 0.7$, but $\bar{C}_{L}$ becomes negative due to the strong suction in the gap side. Sumer \& Fredsøe (1997) reported that if the cylinder is placed in a trench or partially embedded in the sand, the drag force may experience a significant drop, only because the cylinder is protected against the incoming flow. This sheltering effects of the seabed is believed to be significant to the fatigue and stability subsea cables, which have been identified as main areas of concern for marine 
renewable energy projects (Petruneac 2015). Petruneac (2015) innovatively carried out numerical and experimental tests to analyze the turbulence flow features above a seabed profile, scaling down from data of a real seafloor. The research is believed to be useful in optimizing the cable routing.

Griffiths et al. (2012) carried out a parametric study by 2D and three-dimensional (3D) numerical simulations to investigate the hydrodynamic forces on a pipeline with the influence of uneven seabed under current only, wave only and combined conditions. A set of geometric coefficients defining the elevations of the seabed and the pipe was explored. In the 3D study, both partially embedded/spanning pipelines and inclined attack angles were considered, albeit only considering a single isolated span. The spanwise variation of the hydrodynamic loads was quantified. Tong \& Cheng (2016) carried out 3D Large Eddy Simulations (LES) of steady flow around a pipeline very close to an uneven seabed $(G=0.02)$ with staggered gaps underneath the pipe. It was found that the sectional forces vary along the span and oscillate energetically.

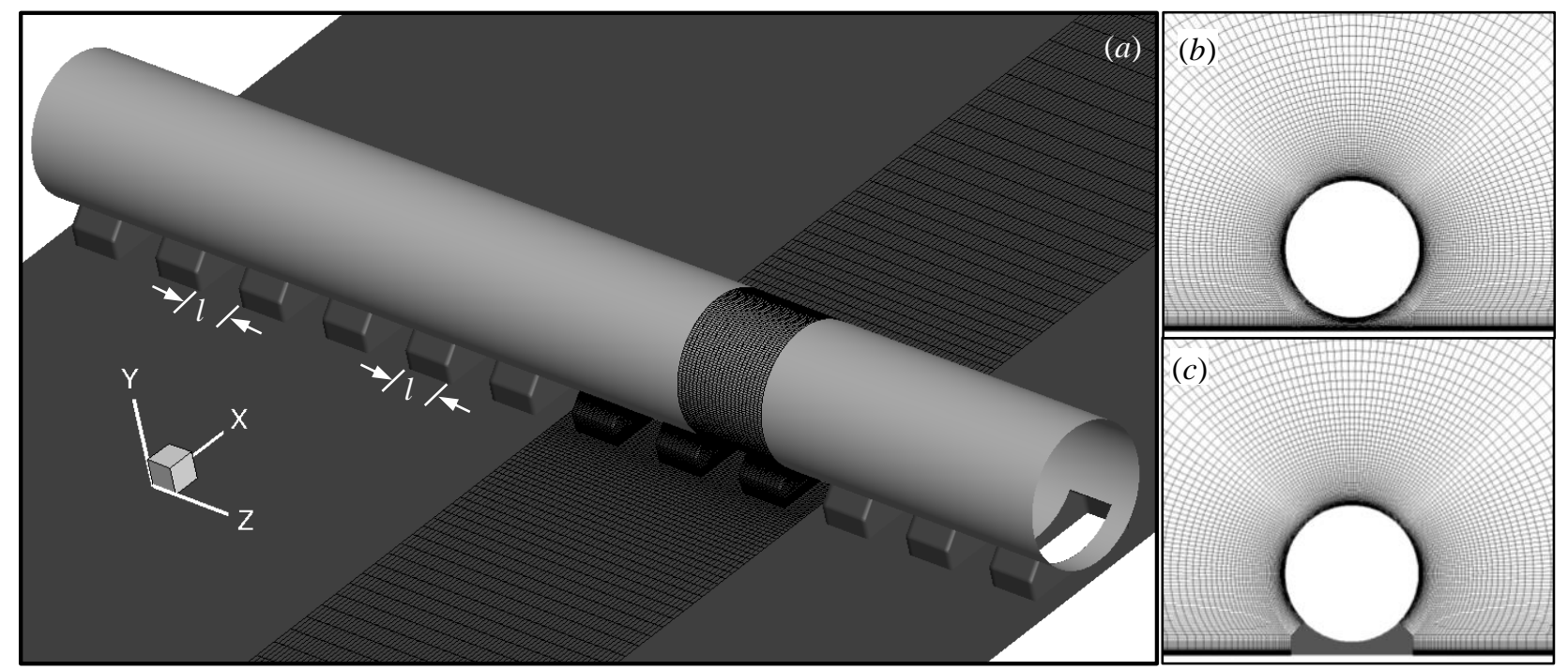

Figure 1 (a), Three-dimensional view of the cylinder (light grey) seated on a wall (dark grey) with staggered gaps. Part of the used mesh is given for the wall, the wedge and the cylinder surface. The pipe elevations above the far-field seabed shown here is $0.1 D$. The meshes used are shown for spanning section $(b)$, and non-spanning contact section $(c)$. The inlet flow is in $x$-direction, from bottom left to top right in $(a)$ and from left to right in $(b)$ and $(c)$.

This paper studies the hydrodynamic forces on a circular cylinder in steady flow seated on an uneven wall, which were mimicked by staggered gaps and wedge-shaped shoulders underneath the cylinder, as shown Figure $1(a)$. A three-dimensional Large Eddy Simulation (LES) was carried out at a fixed $R e=2.5 \times 10^{4}$ and a fixed spanwise length at $9 D$. Three different pipe elevations above the far-field seabed $(G=0.02,0.1$ and 0.2$)$ and three section-length ratios were considered $(L=l / D=$ $0.375,0.75$ and 1.5 , where sectional length $l$ is defined in Figure 1, $a$ ). The aim is to investigate the effects of spanwise-geometry variation, due to rocky seabed for instance, to the hydrodynamic forces and flow features around the pipe. The remainder of the paper is organized in the following manner. In $\$ 2$, the governing equations, numerical model and model validations are presented, while the hydrodynamic force and flow field features are discussed in $\S 3$, and conclusions are given in $\S 4$.

\section{Numerical method and model validation}

\section{$2.1 \quad$ Numerical model}

A Large Eddy Simulation (LES) model is employed in this study. The filtered incompressible 
Navier-Stokes (NS) equations together with the continuity equation read,

$$
\begin{gathered}
\frac{\partial \bar{u}_{i}}{\partial t}+\bar{u}_{j} \frac{\partial \bar{u}_{i}}{\partial x_{j}}+\frac{1}{\rho} \frac{\partial \bar{p}}{\partial x_{i}}=\frac{\partial}{\partial x_{j}}\left(\left[v+v_{t}\right] \frac{\partial \bar{u}_{i}}{\partial x_{j}}\right), \\
\frac{\partial \bar{u}_{i}}{\partial x_{i}}=0
\end{gathered}
$$

where the fluid velocity $u_{i}$ is decomposed into a filtered velocity $\left(\bar{u}_{i}\right)$ and a subgrid-scale velocity $\left(u_{i}{ }^{\prime}\right)$ with $\bar{u}_{i}=u_{i}-u_{i}{ }^{\prime}$ (the same operation also applies for pressure) and $v_{\tau}$ is the subgrid-scale turbulent viscosity. A homogeneous dynamic Smagorinsky model was adopted (Smagorinsky 1963; Germano et al. 1991), due to its demonstrated performance in resolving the flow features in nearwall area (Kirkil \& Constantinescu 2012). These equations are solved through the use of the finite volume discretization OpenFOAM ${ }^{\circledR}$, which is a widely used open-source code. The pressurevelocity coupling is achieved following the Pressure Implicit with Splitting of Operators (PISO) method. The convection terms are discretized using the Gauss cubic scheme, while the Laplacian and pressure terms in the momentum equations are discretized using the Gauss linear schemes. The Euler implicit scheme is adopted for the temporal discretization.

A computational domain of rectangular prism is employed $(x \times y \times z=52 D \times 26 D \times 9 D)$, with the cylinder being placed at the half-way location along the $x$-axis ( $26 D$ away from the inlet) and with its spanwise direction aligned to the $z$-axis. The coordinate system is provided in Figure $1(a)$. The initial values for flow velocity and pressure in the whole domain are set to zero. A uniform inlet velocity $\left(U_{\infty}\right)$ with zero turbulence intensity is set on the inlet boundary, and it is observed that the boundary layer is fairly quickly developed after the start of the simulations, similar to Nishino, Roberts \& Zhang (2008). At the top boundary, the velocity gradients in the $y$-direction and the pressure are set to zero. A symmetry boundary condition is applied at the two lateral boundaries that are perpendicular to the cylinder. No-slip boundary condition is adopted on the cylinder surfaces and the wall boundary.

$\begin{array}{ccccccc}\text { Parameter } \backslash \text { Case } & \text { Mesh } 1 & \text { Mesh } 2 & \text { Mesh } 3 & \text { Mesh } 4 & \text { Exp. A } & \text { Exp. B } \\ \Delta & 1 \times 10^{-3} D & 1 \times 10^{-3} D & 1 \times 10^{-3} D & 0.5 \times 10^{-3} D & & \\ y+ & 1.6 & 1.6 & 1.6 & 0.8 & & \\ \text { Circumference cells } & 120 & 160 & 200 & 200 & - & - \\ z \text { - cells } & 92 & 184 & 276 & 368 & - & - \\ \text { Total cells ( in millions) } & 1.81 & 4.35 & 7.60 & 11.12 & - & - \\ \bar{C}_{D} & 1.20 & 1.23 & 1.18 & 1.19 & 1.14 & 1.23 / 1.26 \\ \bar{C}_{L} & 0.11 & 0.11 & 0.10 & 0.10 & 0.08 & 0.19 / 0.15 \\ C_{L} & 0.40 & 0.39 & 0.27 & 0.29 & 0.20 & 0.30 / 0.38 \\ S t & 0.200 & 0.208 & 0.212 & 0.214 & 0.205 & \text { not given }\end{array}$

Table 1 Details of the meshes used in validation tests for a pipeline placed above a smooth plane boundary at $G=0.4$ and $R e=2.5 \times 10^{4}$. The domain size used in the simulations is $x \times y \times z=52 D \times 26 D \times 9 D$, with the cylinder spanwise direction parallel to the $z$-axis and placed in the middle of the domain along $x$-axis. Experimental (Exp.) data are included for comparison. The Exp. A is from Jensen et al. (1990) at $R e=1.0 \times 10^{4} \& \delta / D=2$ (where it is noted that the undisturbed velocity at the axis of the pipe was used in Equation (1-1) in calculating the force coefficient), and the Exp. B is from Lei et al. (1999) at $R e=1.36 \times 10^{4} \& \delta / D=0.14 / 1.31 \times 10^{4} \& 0.48$.

\subsection{Mesh dependency check}

The size of the computational domain is determined based on experience from previous numerical studies on similar problems (Zhao, Cheng \& Teng 2007). In the spanwise direction, a length of 9D was chosen. In fact, it was found that a spanwise length of $\pi D$ is sufficient in predicting 
hydrodynamic forces at the presently considered $R e$ (Lei, Cheng \& Kavanagh 2001). As shown in Table 1, by changing the mesh sizes and the progression rates of the cell size away from the walls (cylinder surface and plane boundary), four cases were studied with different mesh density, namely the mesh 1 to mesh 4 . For all meshes, the non-dimensional distances from the first nodal point to the wall surfaces (both cylinder and plane wall), $y^{+}=u_{f} \Delta / v$ are kept to be smaller than 2 , where $\Delta$ is the distance from the no-slip surface and $u_{f}$ is the friction velocity. The time-step in the simulations was chosen to keep the Courant number smaller than 0.5 , which usually means the non-dimensional timestep is $\leq 0.002$. Mesh variations on the circumference and on the spanwise direction ( $z$-cells) are studied, which are respectively doubled and quadrupled from mesh 1 to mesh 4 . The finest mesh has more than 11 million cells in total as shown in Table 1. Typical mesh distribution around the cylinder and wall is given in Figure 1.

A case with $R e=2.5 \times 10^{4}$ and $G=0.4$ was studied in the validation tests. The numerical results on $C_{D}, C_{L}$ and $S t$ are listed in Table 1 . The force coefficients are generally affected little by the change in mesh density among the meshes chosen and they appear to agree well with experimental data from Jensen et al. (1990) and from Lei et al. (1999) for a circular cylinder in the proximity to a smooth wall, especially for the mean drag coefficient $\bar{C}_{D}$ and St. Compared to Jensen et al. (1990), all cases over-predicted the root-mean-square $(r m s)$ lift coefficient $C_{L-r m s}$, but it is consistent with Lei et al. (1999). The mean lift coefficient $\bar{C}_{L}$ falls into the range reported by the two experimental tests. For the vortex shedding frequency, it seems all meshes yield satisfactory results as compared to Jensen $e t$ al. (1990). From these comparisons, the majority of results reported here were based on the mesh 3.

\begin{tabular}{ccc}
\hline$G$ & $L$ & $N_{\text {spanning }} / N_{\text {non-spanning }}$ \\
$0.02,0.1,0.2$ & 0.375 & $13 / 12$ \\
& 0.75 & $7 / 6$ \\
& 1.5 & $4 / 3$ \\
Table 2 The tested zebraic cases. For each sectional length ratio $(L)$, three cylinder elevations above the far-field seabed \\
$(G)$ were considered; while for each $G$, three $L$ were investigated. The numbers of spanning sections $\left(N_{\text {spanning }}\right)$ and non- \\
spanning sections $\left(N_{\text {non-spanning }}\right)$ are also given for each $L$.
\end{tabular}

\section{Result discussions}

As summarized in Table 2, the effects of small spanning on hydrodynamic forces were considered by varying the far-field elevation ratio $G$ and the sectional-length ratio. Three sectional lengths have been considered at $L=0.375,0.75$ and 1.5 for each $G$ ranging from 0.02 to 0.2 . The spanwise lengths of the spanning and non-spanning sections are the same, except at two spanwise ends, where the length of the two gap sections are both kept at $L / 2$ (with symmetry boundary condition applied at two sides of the domain, see Figure $1, a$ ). Therefore, the ratio of spanning has been kept constant at $50 \%$ and this leads to in total 25 (including 13 gap and 12 embedded), 13 (7 gap and 6 embedded) and 7 (4 gap and 3 embedded) sections, respectively, at $L=0.375,0.75$ and 1.5. These cases are referred to as the zebraic case in the following discussion. For comparison, simulations on spanwiseuniform cylinders with $100 \%$ non-spanning and $100 \%$ spanning are also carried out and these cases are named as the all-spanning case and fully non-spanning case, respectively.

Unlike a conventional numerically modelled embedded cylinder, in this work a quarter of the cylinder surface was covered by the wedge-shoulder type of seabed in the present non-spanning scenarios, as illustrated in Figure $1(a, c)$, irrespective of the $G$ ratios. This means that the hydrodynamic forces for all-spanning, zebraic and fully non-spanning are directly comparable since the elevation of the cylinder compared to the far-field seabed is not changing and only the geometry of the local seabed immediately under the pipe is changing. Unless otherwise specified, both the 
seabed wall and the wedge shoulder have been treated as impermeable, which means the cylinder contacted area has been cut off in calculating the forces. As noted by Jacobsen et al. (1989) and An et al. (2011), this approach would lead to smaller in-line forces. As compared to a rounded shape, the adopted sharp-edged shoulder is known to complicate the flow patterns around structures (Tian et al. 2014) and increase the flow kinetic energy. However, a rounded shoulder or even elastic foundation (Sollund, Vedeld \& Fyrileiv 2015) is worthwhile for future investigations.

It should also be noted that the unaffected boundary layer thickness $\delta / D$ at the cylinder location (without the cylinder) is 0.16 in the present study and this was kept constant for all simulations. The $\delta / D$ has been demonstrated as a significant factor influencing the hydrodynamic forces on the cylinder (Lei et al. 1999).

\subsection{Spanwise uniform cylinder}

Figure 2 and Figure 3 present the force coefficient on the spanwise-uniform cylinder, providing a reference for comparison with that of the zebraic case later on. The forces in Lei et al. (1999) obtained from experiments for a circular cylinder close to a smooth bed are also included in Figure 2. For the all-spanning case, the $\bar{C}_{D}$ increases about $10 \%$ with the increase of $G$ from 0.02 to 0.2 , while the $\bar{C}_{L}$ is more than halved, dropping from 0.42 to 0.17 . Both $\bar{C}_{D}$ and $\bar{C}_{L}$ generally fall into the range of experimental data, despite the difference in $R e$.

For the fully non-spanning case as in the present setting, both $\bar{C}_{D}$ and $\bar{C}_{L}$ as given in Figure 3 appear to be only slightly affected by the change of $G$. It is noted that at the largest $G$ ratio $(0.2)$, the cylinder would be totally outside of the undisturbed boundary layer. This result appears to suggest that the influence of $G$ ratio can be neglected in the $100 \%$ non-spanning case.

(a)

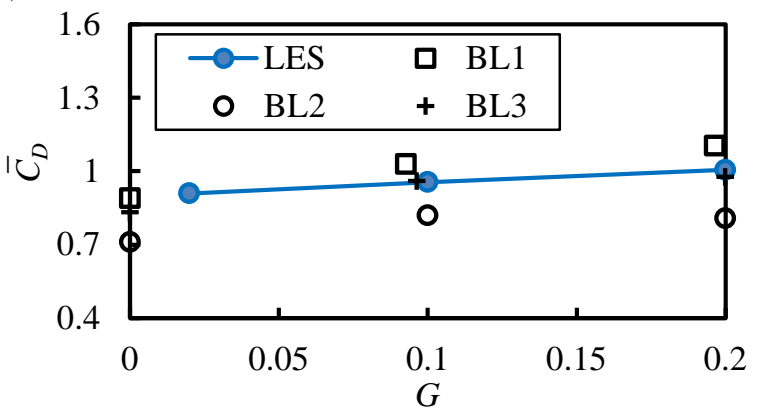

(b)

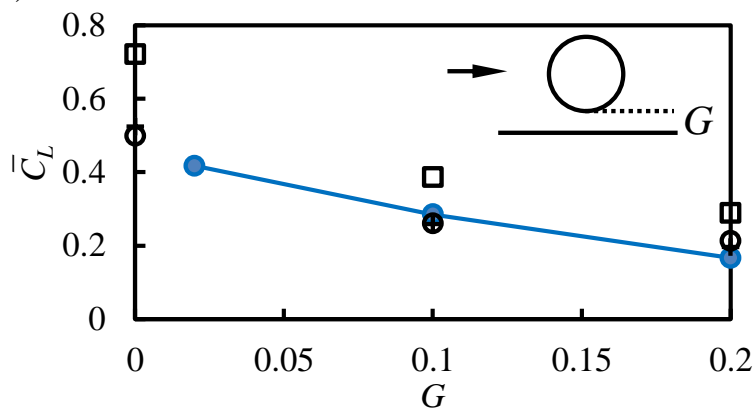

Figure 2 Comparison on the drag $(a)$ and lift $(b)$ coefficients as a function of $G$ for the all-spanning case (smooth wall) from present LES simulations with experimental study by Lei et al. (1999). BL1, BL2 and BL3 represent data from three settings in the lab tests, where $\delta / D(R e)$ are $0.14\left(1.36 \times 10^{4}\right), 0.25\left(1.38 \times 10^{4}\right)$ and $0.48\left(1.31 \times 10^{4}\right)$, respectively.

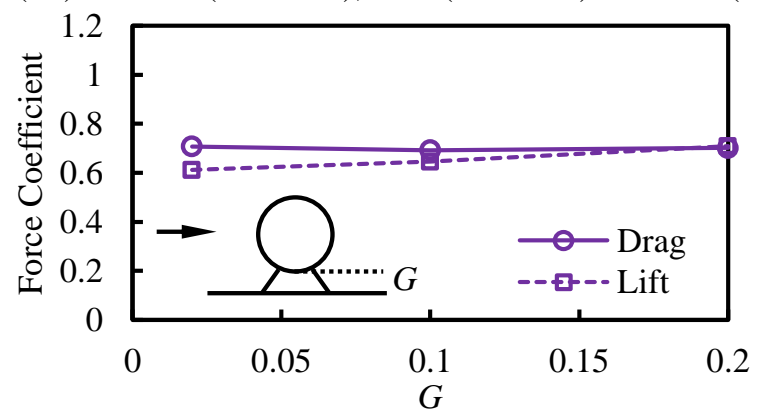

Figure 3 Force coefficients as a function of $G$ for the fully non-spanning case.

\subsection{Sectional forces for zebraic-contact cylinders}

The statistics on force coefficients of each section from the zebraic cases at three $G=0.1$ are given in Figure 4 as bar plots, including the $\bar{C}_{D}, \bar{C}_{L}$ and the rms values, along with the corresponding 
results over the whole cylinder in the zebraic (red bars), all-spanning (blue lines) and fully nonspanning (purple lines) cases. The influence of $L$ to the sectional forces is mainly discussed with reference to the case with $G=0.1$ in Figure 4 and similar features are also observed at $G=0.02$ and 0.2 . Three main features in the force statistics can be observed.

a) The $\bar{C}_{L}$ (Figure $4, a$ ) on the spanning sections and non-spanning sections are respectively close to the corresponding spanwise uniform cases. The $\bar{C}_{L}$ on the spanning sections only slightly increases with the decrease of $L$. This suggests $L$ has limited influence on sectional $\bar{C}_{L}$ and thus the total lift over the cylinder with zebraic gaps underneath is proportional to the ratio of spanning/non-spanning sections. With $50 \%$ spanning ratio, the sectional $\bar{C}_{L}$ (the red bar) is within the range of $0.45 \pm 0.3$, which is very close to the average (0.46) of the all-spanning case (0.26) and the fully non-spanning case (0.65).

b) The $\bar{C}_{D}$ (Figure $4, b$ ) on non-spanning sections are about $15 \%$ larger than that of the fully nonspanning case, while on spanning sections $\bar{C}_{D}$ are $10 \%$ smaller than the all-spanning case. The difference in $\bar{C}_{D}$ between non-spanning sections and the corresponding spanwise-uniform case appears to increase with the decrease of the $L$. Similar to $\bar{C}_{L}$, the total $\bar{C}_{D}$ of the zebraic case can be estimated as the average of $\bar{C}_{D}$ of spanwise-uniform cylinders.

c) The sectional length has the most noticeable influence on oscillation amplitudes of forces (Figure 4, $c$ and $d$ ), which can be 4 times as large as their counterparts for spanwise uniform cases. Interestingly, the energetic oscillations in the sectional forces do not lead to large amplitude of oscillations in the total force over the whole cylinder, which can be even smaller than that of the all-spanning cases. As it will be seen later on, this is mainly due to the phase difference in the time history of the forces.

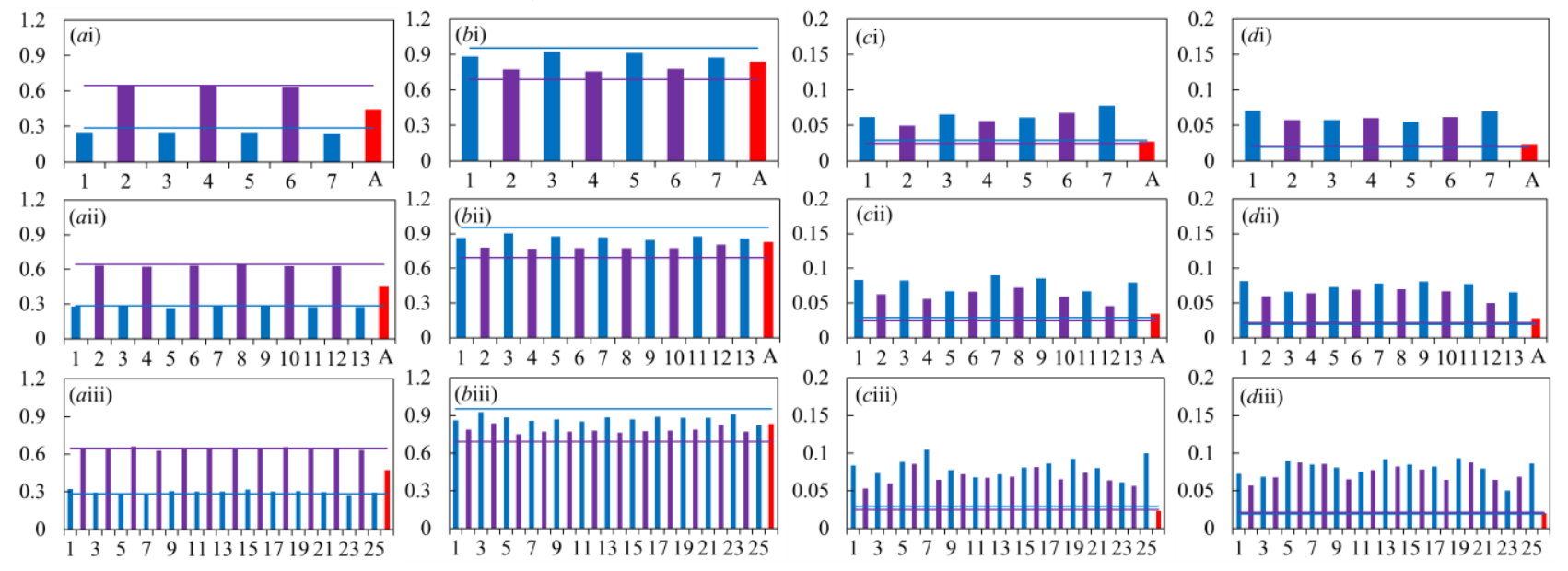

Figure 4 Force coefficients at $G=0.1$ of the cylinder on an uneven wall with intermittent contact. From left to right are the mean lift coefficient $\left(\bar{C}_{L}, a\right)$, mean drag coefficient $\left(\bar{C}_{D}, b\right)$,rms lift coefficient $\left(C_{L-r m s}, c\right)$ and $r m s$ drag coefficient $\left(C_{D-r m s}, d\right)$. The blue bars represent the force coefficient on each gap section, purple ones on each embedded section, and the red bars the correspondent statistics on the cylinder as a whole. The sectional lengths from top to bottom are (i) $1.5 \mathrm{D}$, (ii) $0.75 \mathrm{D}$ and (iii) $0.375 \mathrm{D}$. Blue and purple lines correspond to the statistics of the $100 \%$ all-spanning case and $100 \%$ fully non-spanning case.

\subsection{Influences of $G$ and $L$}

Figure 5 and Figure 6 show the differences between mean sectional forces and their counterparts of spanwise-uniform cylinders, to illustrate the effects of cylinder elevation $G$ ratio and zebraic sectional length $(L)$ on the hydrodynamic forces. The Difference in forces is calculated by 


$$
\text { Difference }=\frac{\sum_{i=1}^{N} \frac{C_{X}^{i}}{N}-C_{X}}{C_{X}},
$$

where $C_{X}$ is either $\bar{C}_{D}$ or $\bar{C}_{L}$ from the spanwise-uniform cylinders, $C_{X}^{i}$ is the corresponding mean force coefficient on the $i$-section in the zebraic case, and $N$ is the number of sections.

(a)

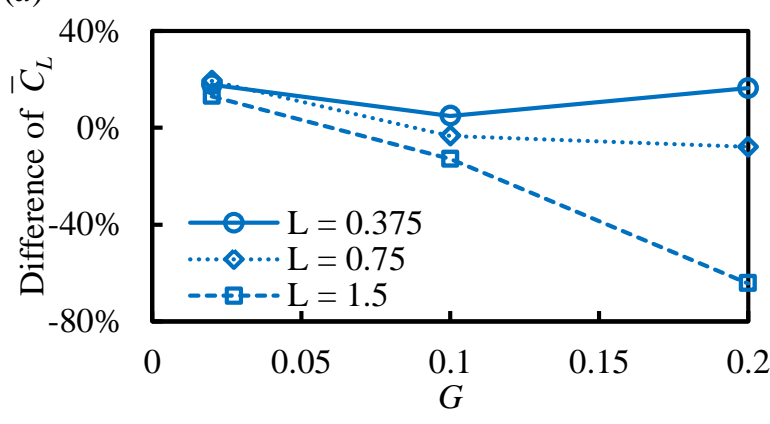

(c)

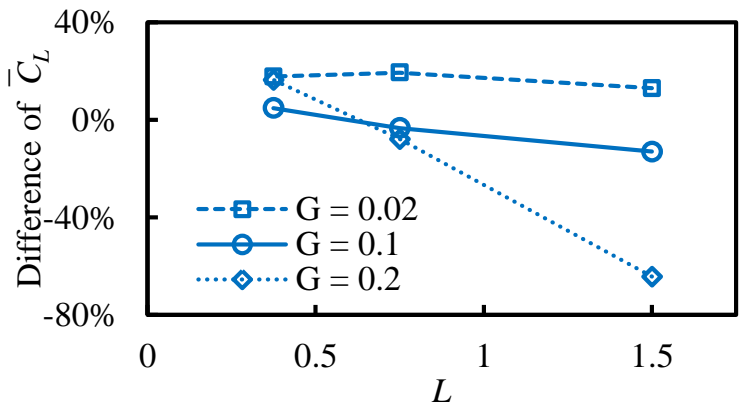

(b)

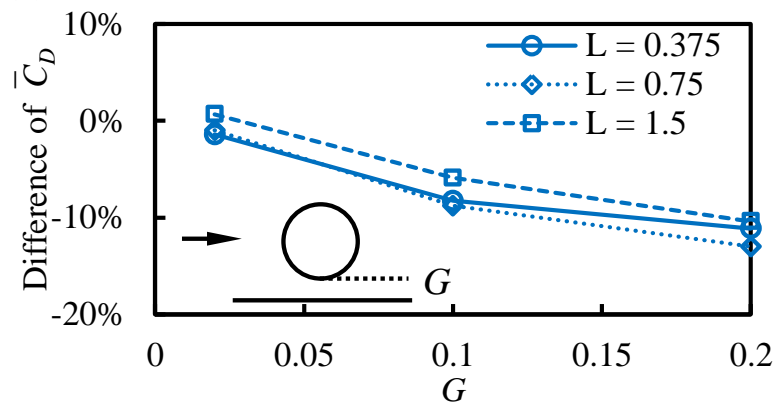

(d)

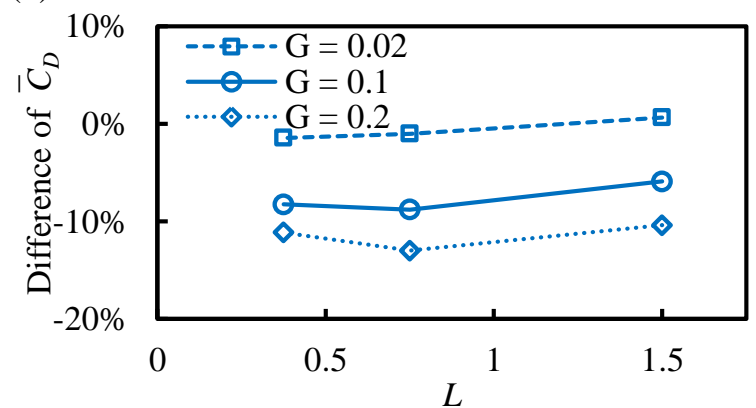

Figure 5 The Difference in mean force coefficient on the spanning sections as compared to that of the $100 \%$ all-spanning case, calculated from equation (3-1). (a) and (b) show the influence of $G$ ratio, while $(c)$ and $(d)$ highlight the effect of $L$.

(a)

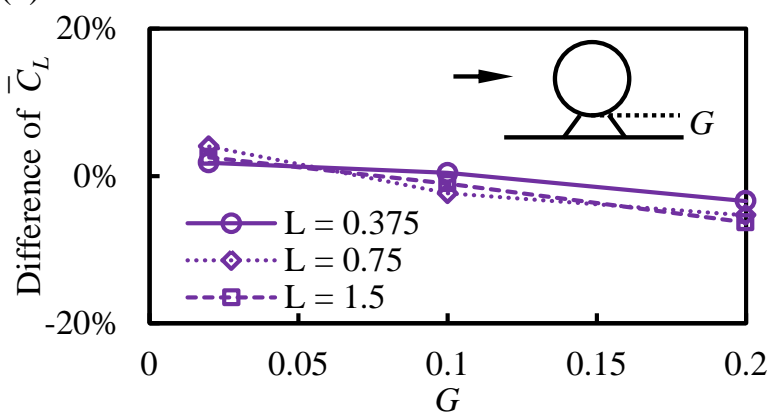

(c)

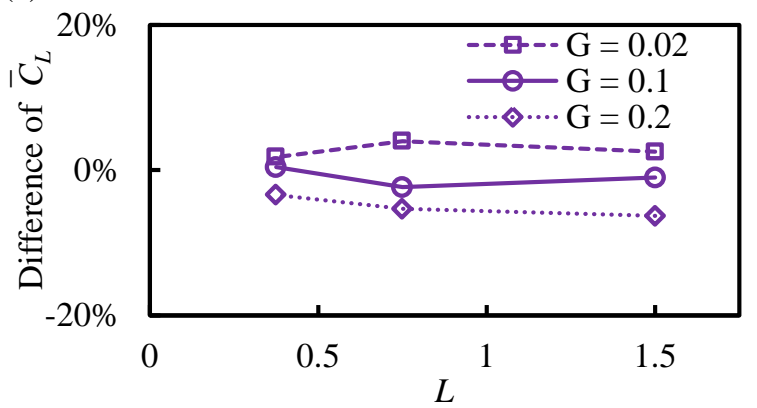

(b)

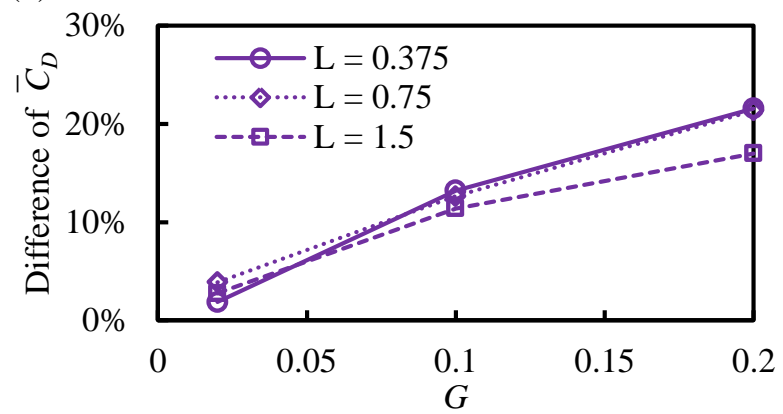

(d)

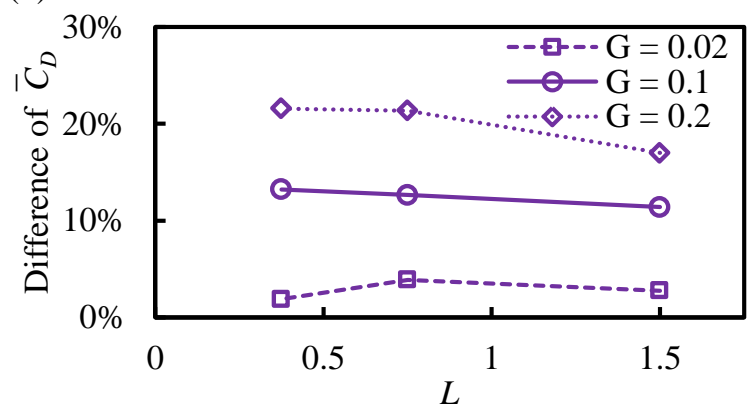

Figure 6 The Difference in mean force coefficient on the non-spanning sections as compared to that of the $100 \%$ fully non-spanning case, calculated from equation (3-1). (a) and $(b)$ show the influence of $G$ ratio, while $(c)$ and $(d)$ highlight the effect of $L$. 
For the lift coefficient on the spanning sections, Figure $5(a)$ and $(c)$ illustrate the same set of data against $G$ and $L$, respectively. The Difference of $\bar{C}_{L}$ is within $20 \%$ at the two small $L$ or $G$; however, it enlarges significantly with the increase of $G$ at $L=1.5$ (Figure 5, $a$ ). The sectional $\bar{C}_{L}$ can be more than $60 \%$ smaller than that of the all-spanning case at $(L, G)=(1.5,0.2)$, where the relatively large spans (both spanwise and vertically) are believed to enhance gap flow (deflected from the nonspanning section to the spanning section as shown later in section 3.6) and this contributes to a low pressure on the bottom surface of the cylinder in the spans.

On the other hand, the change of $G$ and $L$ only brings less than $20 \%$ difference on sectional $\bar{C}_{D}$. Once again, the largest difference was found at the largest $G$ and $L$. Figure $5(b)$ and $(d)$ also show that the Difference of $\bar{C}_{D}$ is less influenced by the change of $L$ at a fixed $G$, but it decreases steadily with the increase of $G$ at a fixed $L$, in agreement with the trend in the all-spanning case (Figure 2).

Figure 6 illustrates the similar information as in Figure 5 but for the non-spanning sections. With the change of $G$ and $L$, the Difference in $\bar{C}_{L}$ is less than $10 \%$ as compared to the fully non-spanning case, while the Difference in $\bar{C}_{D}$ is less than approximate $20 \%$. Since Figure 3 has already shown that the force coefficient in the fully non-spanning case is generally independent of $G$ ratio, the change of $\bar{C}_{D}$ or $\bar{C}_{L}$ in Figure 6 is very likely due to the deflected flow from non-spanning sections to the spans, and this is the reason that the most significant change is observed at the largest $G$ and $L$.

(a)

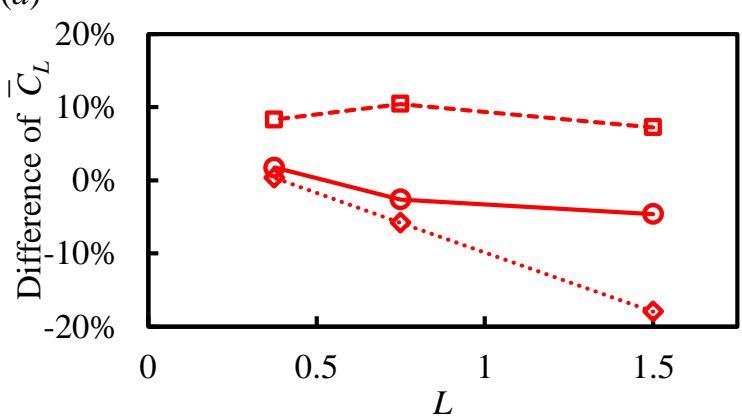

(b)

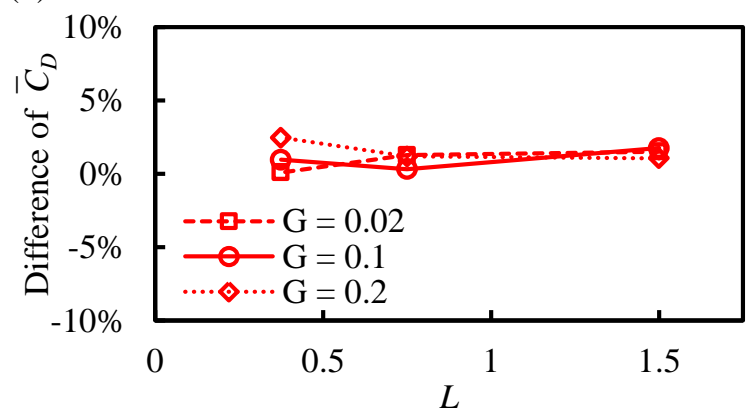

Figure 7 Total mean force coefficient from the numerical simulation of the zebraic case as compared to that predicated from equation (3-2).

From the above observations, the total hydrodynamic forces for a circular cylinder seated on an uneven seabed with zebraic contact can be approximately calculated by independently considering the spanning sections and the non-spanning sections. That is, the force coefficient can be calculated by a weighted average of the forces from the spanwise-uniform cylinder, according to,

$$
C_{X}^{T}=0.5 C_{X}^{s}+0.5 C_{X}^{n},
$$

where $C_{X}^{T}$ is the total force coefficient of the zebraic case (either $\bar{C}_{D}$ or $\bar{C}_{L}$ ), $C_{X}^{s}$ and $C_{X}^{n}$ are the force coefficient from the spanwise-uniform cases (all-spanning / fully $\boldsymbol{n}$ on-spanning cases), and 0.5 is the spanning ratio in the present study. Note that this conclusion warrants further investigation and confirmation at different ratios of spanning. Figure 7 shows the difference of total force coefficient from the zebraic cases in the simulations (as given by red in Figure 4) with that calculated from equation (3-2). It is seen that the equation predicts less than $5 \%$ difference in $\bar{C}_{D}$, while less than $20 \%$ in $\bar{C}_{L}$. For $\bar{C}_{L}$, equation (3-2) performs best at $G=0.1$, while it under-predicts about $10 \%$ at $G=$ 0.02. Again, the largest difference in $\bar{C}_{L}$ is found at $(L, G)=(1.5,0.2)$, where equation (3-2) overpredicts $\bar{C}_{L}$ for approximate $20 \%$. This is believed to be due to the deflection of flow from nonspanning to spanning sections as discussed in Figure 5. This size difference also suggests that the 
zebraic setting tends to discourage gap flow at low $G$ ratio (thus the lift increases compared to the all-spanning case at the same $G$ ), while it encourages gap flow at large $G$ ratio (thus the lift decreases).

\subsection{Resolved hydrodynamic force direction}

The direction of resolved hydrodynamic force is calculated based on the total $\bar{C}_{D}$ and $\bar{C}_{L}$ across the whole cylinder for the zebraic cases as well as the spanwise-uniform cases. Figure 8 shows the variation of force direction as a function of $G$ for the five different geometry settings. For the fully non-spanning cylinder, the the resolved direction appears to be indepdent of $G$, only showing $5^{\circ}$ of rise from about $40^{\circ}$ as $G$ increases from 0.02 to 0.2 . For the fully spanning cylinder, the rotation angle significantly decreases from $25^{\circ}$ to less than $10^{\circ}$ in the range of $G$. For the three zebraic cases, the rational angle all shows a trend of drop, while the value falls between that of the two spanwiseuniform cylinders. The rotaitonal angle indicates the relative size of $\bar{C}_{L}$ as compared to $\bar{C}_{D}$, as such for large angles $\left(45^{\circ}\right)$, the lift force is comparable to the drag, while for small angles, the lift force is smaller than the drag.

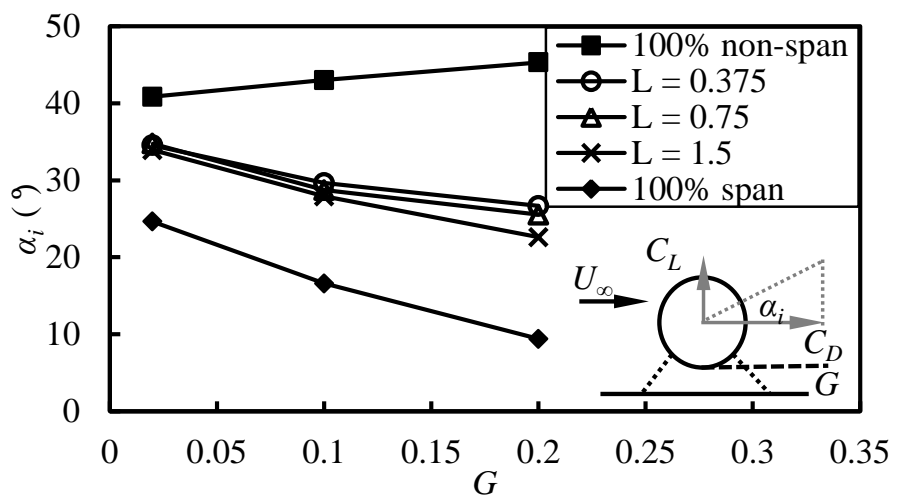

Figure 8 The resolved direction of hydrodynamic forces calculated based on the total mean forces on the cylinder as a whole. Here the zebraic cases are represented by the sectional length ratio $(L)$.

\subsection{Time history of forces}

The time histories of the sectional force coefficients are presented in Figure 9 for two selected sections from the zebraic case at $G=0.1$. The $C_{L}$ oscillates above zero at $G=0.1$, leading to a repelling force from the wall. This is mainly due to the influence of wall proximity on pressure distribution on the surface of the cylinder. In the extreme case, the $C_{L}$ is comparable to $C_{D}$ for the non-spanning sections. However, with the increase of cylinder elevation ratio to $G=0.2$, the $C_{L}$ oscillates at a lower average value.

The phase of the force coefficients over spanning and non-spanning sections keeps changing between in-phase and anti-phase, as illustrated with two boxes at Figure 9 (i); partially due to this feature, the rms values of the total force coefficient are smaller than that of sectional forces, in a similar way to the difference between sectional force coefficients and total force coefficients for an isolated cylinder, as observed by Zhao, Cheng \& Zhou (2009).

Another reason for the large amplitude of oscillation in sectional forces is due to the gap flow. The fluid near the non-spanning sections is partially deflected to the spanning sections in order to pass the cylinder, and this is not expected to be a steady process. Figure 10 illustrates the vector forms of the gap flow at $L=1.5$ and $G=0.1$. The velocity vector is the volume-averaged velocity, calculated by integrating the respective velocity component over a volume of half of the span gap (from the shoulder to the middle of the span in the spanwise direction). The volume-averaged spanwise velocity $\left(U_{z}\right)$ goes up and down frequently, suggesting the change in directions and magnitudes of 
the gap flow, and it certainly is expected to bring in changes to the force coefficient. The volumeaveraged wall normal velocity $\left(U_{y}\right)$ also changes directions, but less frequently and mostly it directs towards the wall.

The variations in the time history of force coefficients in Figure $9(a, b)$ and the gap flow in Figure 10 are due to the turbulent flow structures, rather than vortex shedding, which is not observed in all zebraic cases in the present study. This is evidenced by the Fast Fourier transform (FFT) of the lift coefficient on section 5 (Figure 9,c). No apparent dominant peaks at any particular frequency are prominent, suggesting no coherent vortex shedding. Similar FFT spectra were also observed for the non-spanning sections.
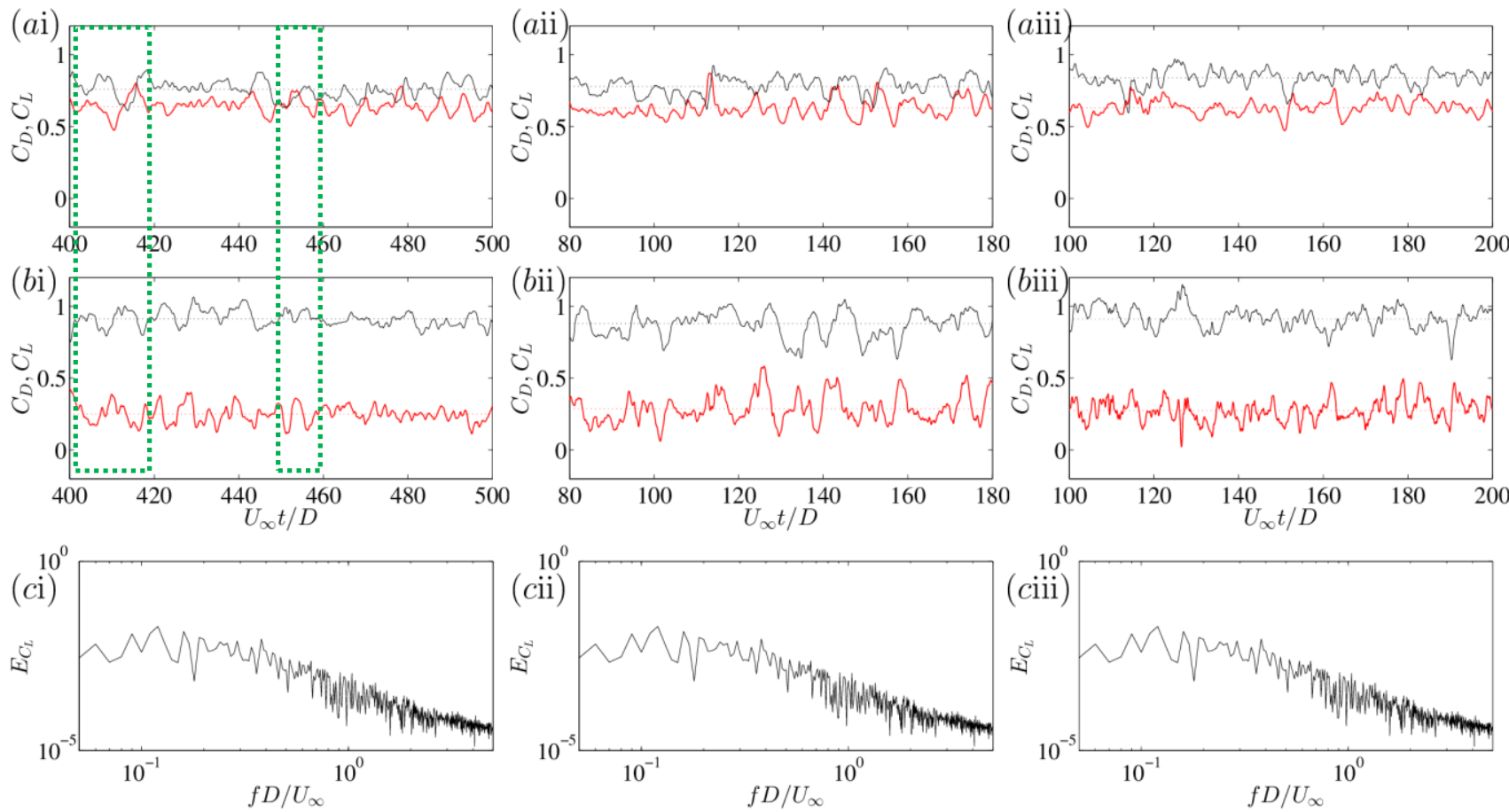

Figure 9 Time evolution of the drag coefficient $\left(C_{D}\right.$, thin black) and lift coefficient $\left(C_{L}\right.$, thick red) from $G=0.1$ on a selected non-spanning section ( $a$, section 5 , as given in Figure 4$)$ and spanning section ( $b$, section 4 , also see Figure 4$)$ for sectional length at (i), $1.5 \mathrm{D}$; (ii); $0.75 \mathrm{D}$; and (iii), $0.375 \mathrm{D}$; The dotted lines represent the mean values. The FFT on lift coefficient on section 4 is given in (c).

(a)

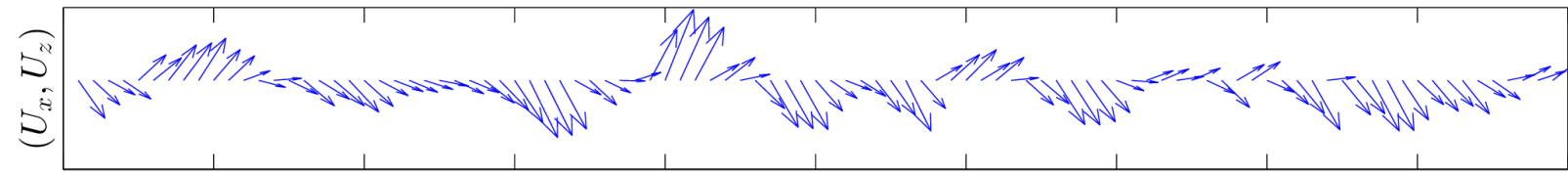

(b)

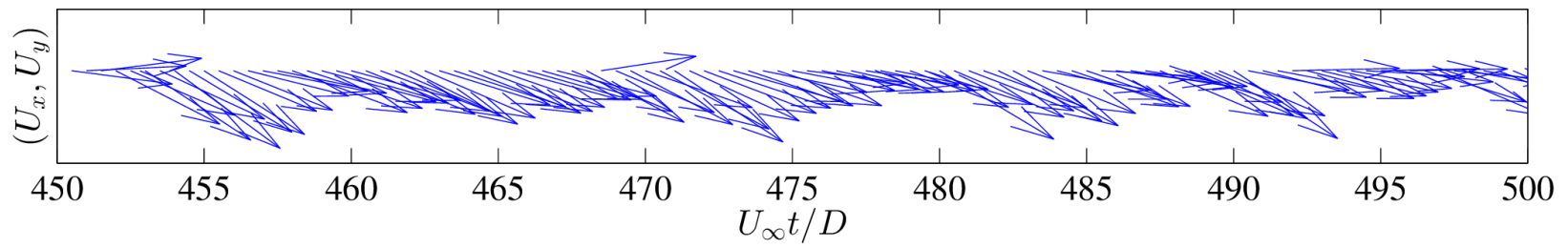

Figure $10 \mathrm{Gap}$ velocity vector at $G=0.1$ and $L=1.5 \mathrm{D}$. Samples were made over every 0.5 non-dimensional time $\left(U_{\infty} t / D\right)$. At any sampling point, the velocity components were volume-averaged over half of the gap in the spanwise direction.

\subsection{Flow fields}

Figure 11 shows the flow field as represented by the $\lambda_{2}$-definition (Jeong \& Hussain 1995; Tong, Cheng \& Zhao 2015) at the medium $G$ ratio, colored by instant velocity in the $x$-axis direction. It is observed that the wall boundary layer is dominated by turbulent flow structures in front of the 
cylinder. However, no noticeable changes in the flow field can be easily observed with the change of $L$, in agreement with the observations in the hydrodynamic forces at this case. This is because the gap flow only affects the field very close to the seabed below the cylinder. The sectional difference in mean force coefficient and the large oscillation amplitude of sectional forces, as shown in Figure 4, can be partially explained by the spanwise variations in the flow field across the whole cylinder and the hairpin type of turbulent flow structures in front of the cylinder (in the shape of $\Lambda$ ).
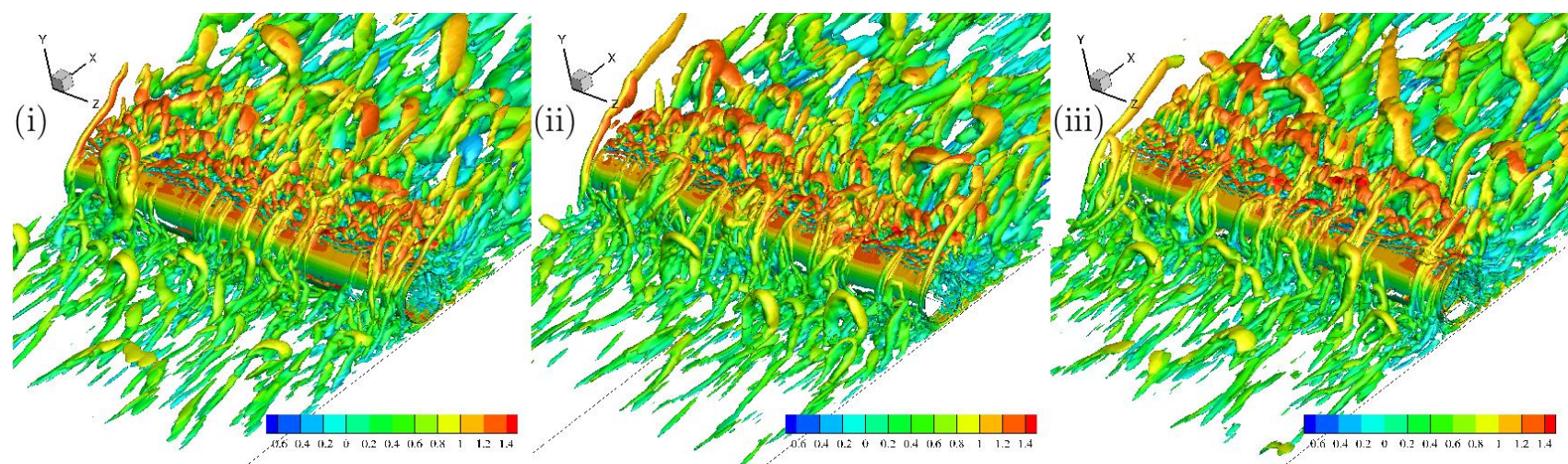

Figure 11 Flow field as given by $\lambda_{2}$-definition $(=-1)$, colored by instant $U_{x}$ for sectional length at $1.5 D$ (i), $0.75 D$ (ii) and $0.375 D$ (iii) at $G=0.1$.

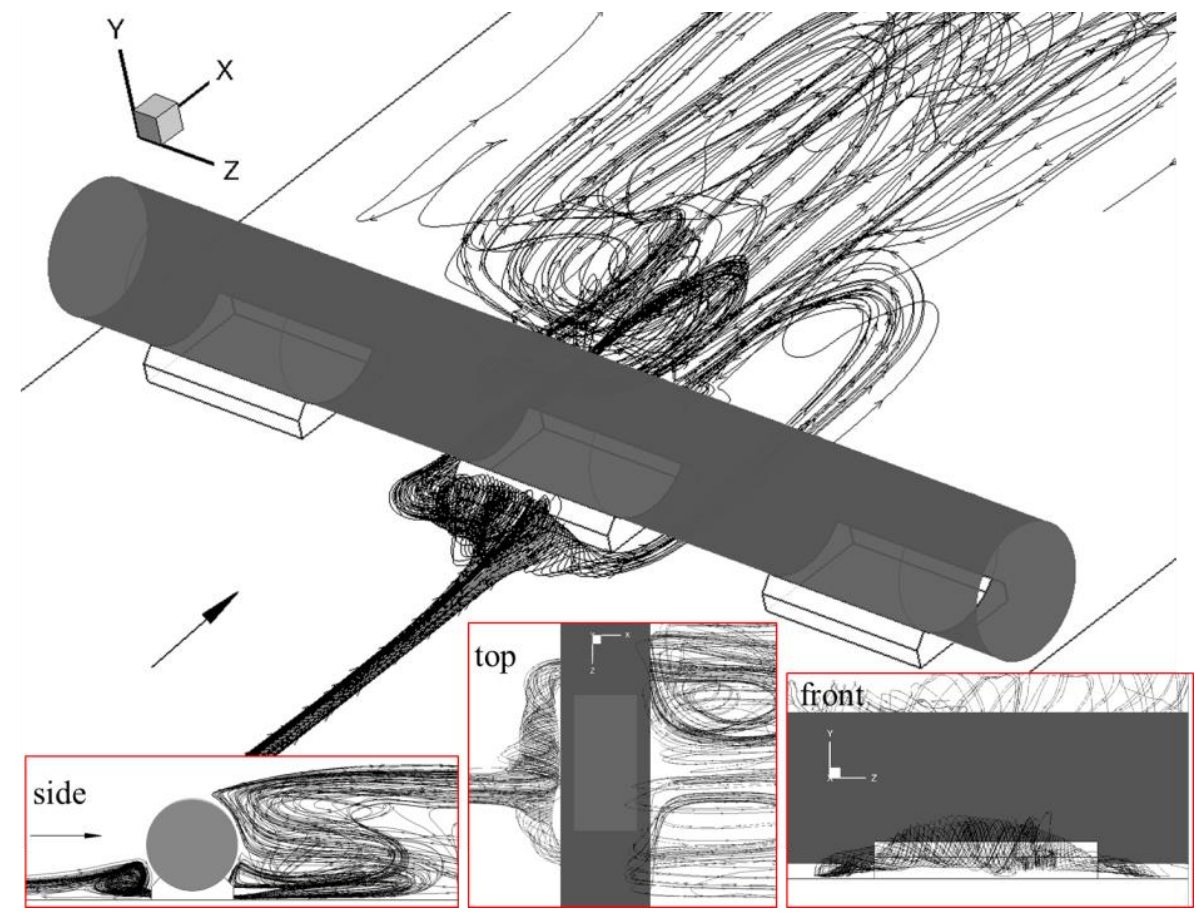

Figure 12 Streamlines based on the averaged flow field at $G=0.1$ and $L=1.5$. In total 50 streamlines were generated along a line parallel to the cylinder in front of the non-spanning section at the same height of the bottom of the cylinder. The three inserts show the same streamlines from different side of view.

Figure 12 shows an overview of the streamlines from the averaged flow field at $G=0.1$ and $L=$ 1.5. The deflection of the fluid from the non-spanning section to spanning section is obvious in this case, where the streamline spins as a spanwise vortex in front of the cylinder, apparently due to the blockage effect of the wedge shoulders. The streamlines are then being sucked through two neighboring gaps and forced to swirl after passing over the cylinder in the wake. This type of gap flow, as seen from the change in forces, is slightly weakened at $(G, L)=(0.02,0.375)$, but enforced at $(G, L)=(0.2,1.5)$. 

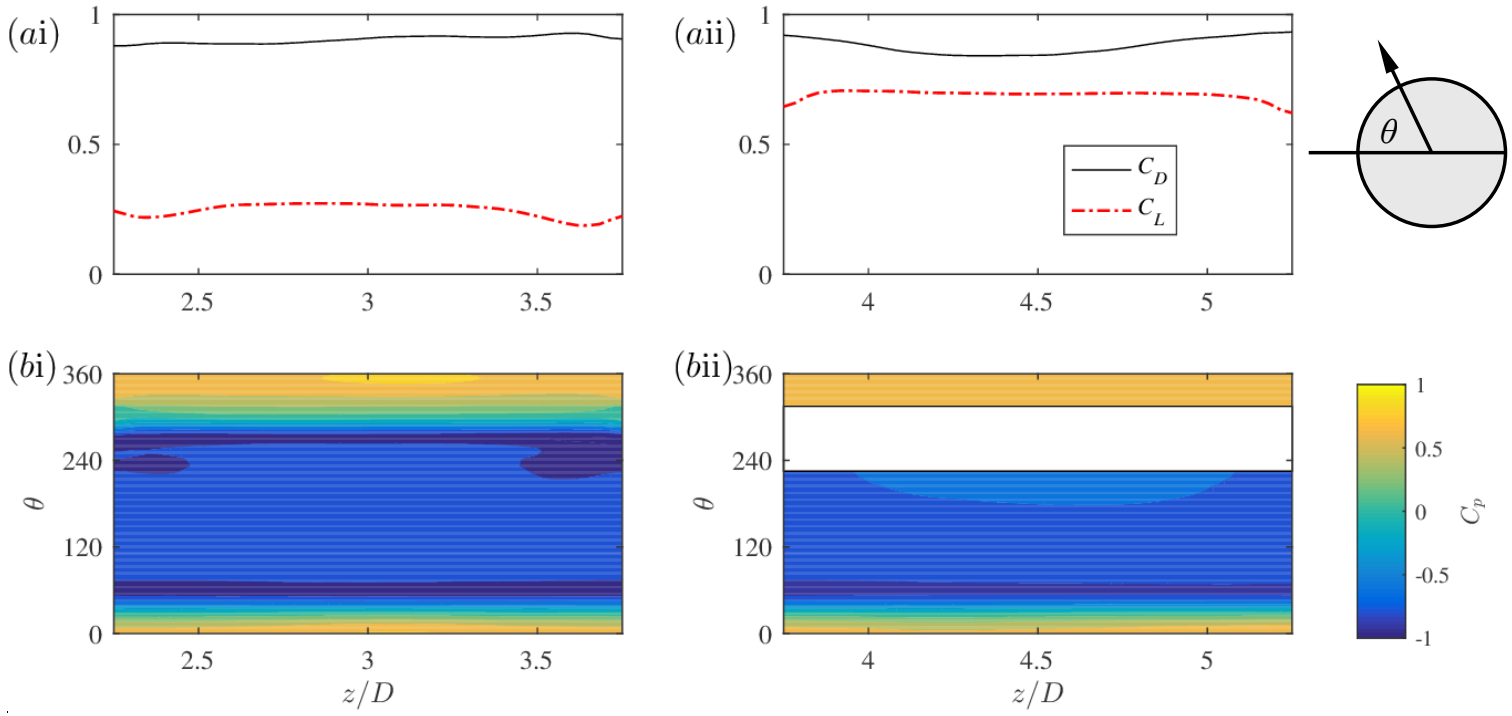

Figure 13 The change of (a) pressure drag coefficient $\left(C_{D}\right.$, solid line) and lift coefficient $\left(C_{L}\right.$, dashed line) on the (i) gap section and (ii) embedded section (in front of which streamlines were shown in Figure 12). $C_{D}$ and $C_{L}$ were integrated from the $(b)$ pressure distribution on cylinder surface at $G=0.1$ and $L=1.5$.

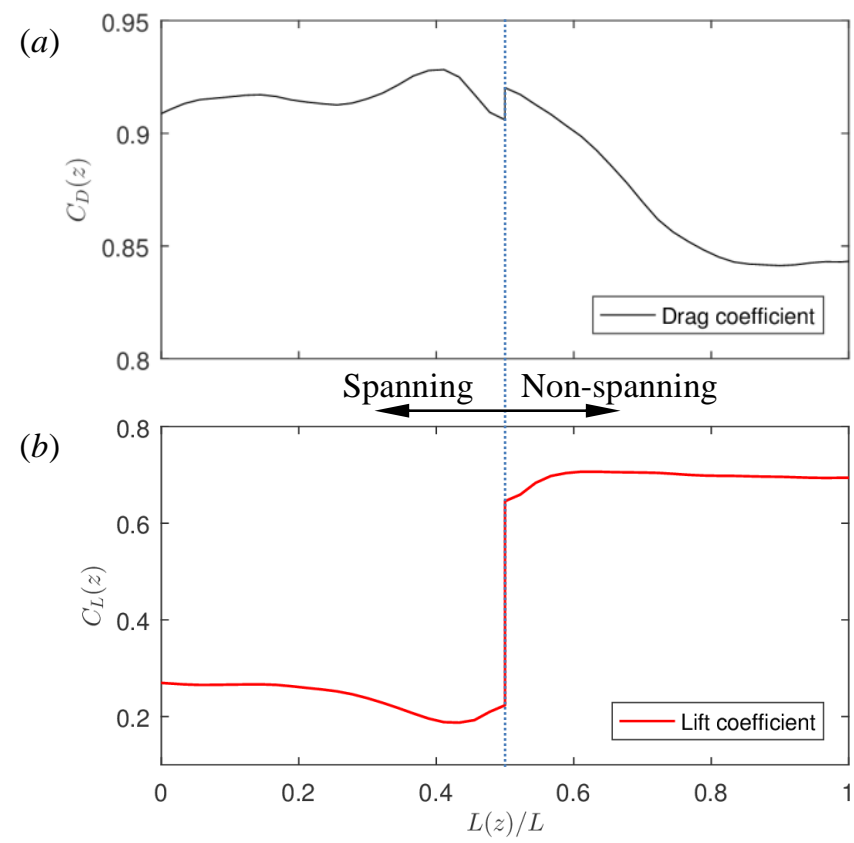

Figure 14 The sectional force coefficients along the span of the cylinder at $R e=2.5 \times 10^{4}, G=0.1$ and $L=1.5$, focusing on the transition from spanning section to non-spanning section. Here, $L(z) / L=0$ corresponds to mid-span location, while $L(z) / L=1$ to mid-non-spanning location. Thus, $L(z) / L=0.5$ is the transition point.

Figure 13 quantitatively illustrates how the gap flow affects the distribution of pressure coefficient $\left(C_{p}\right)$ on different sections at $(G, L)=(0.1,1.5)$. Here, the $C_{p}$ is defined as,

$$
C_{p}=\frac{p(z, \theta)-p_{\infty}}{0.5 \rho U_{\infty}^{2}}
$$

where $p_{\infty}$ is the far-field pressure and $p(z, \theta)$ is the averaged pressure on the surface of the cylinder and $\theta$ is defined in Figure 13. It is seen the surface $C_{p}$ inside the gap (approximately $220^{\circ}<\theta<320^{\circ}$ ) or near the wedge varies greatly, while the $C_{p}$ on the front surface, facing the incoming flow, is less affected by the geometry difference. For the spanning section in Figure 13 (bi), the peak negative 
pressure was found where the deflective streamlines passing through, as a results the integrated $C_{L}$ dips at $z / D=2.4$ and 3.6. For the non-spanning section in Figure 13 (bii), the surface pressure in the middle of the downstream wedge is slightly increased and the integrated $C_{D}$ decreases accordingly. Generally, the $C_{L}$ on the spanning section is most influenced by the spanwise-geometry difference.

The change in force coefficient along the span of the cylinder is shown Figure 14 for the above case among $z / D=3 \sim 4.5$, focusing on the floor configuration transitions from spanning to nonspanning. Considering in engineering applications where the seabed is porous or permeable, here $C_{D}(z)$ and $C_{L}(z)$ are calculated from the mean pressure distribution on the surface of the cylinder and a linear interpolation of the surface pressure on the embedded section $\left(225^{\circ}<\theta<315^{\circ}\right)$. As discussed earlier, this approach is different from that in computing the forces for all above discussion, where the bed has been assumed impermeable and the pressure on the embedded surface was excluded.

A significant jump in $C_{L}(z)$ is observed in Figure 14 as the configuration changes from spanning to non-spanning (from about 0.2 to 0.7 ), highlighting the influence of the different configurations. The lift coefficient is also found to vary slightly as it moves from mid-span to mid-embedded due to the flow diversion as it passes the cylinder. The $C_{D}(z)$ is less affected compared with $C_{L}(z)$, and only very small discontinues were observed at the transition point. This observation is generally consistent with those reported by Griffiths et al. (2012).

\section{Conclusions}

We present a numerical study on the steady flow around wall-bounded cylinder by Large Eddy Simulation. The hydrodynamic forces have been investigated under the influences of small intermittent spanning and non-spanning sections across the spanwise direction of the cylinders. Three pipe elevation ratios between the cylinder and the far-field wall $(G)$ are considered, along with three sectional-length ratios $(L)$ at each $G$. The main findings are summarized as follows,

1) The spanwise difference brings distinct changes to the sectional forces. The drag coefficient on spanning sections is generally smaller than the corresponding spanwise-uniform cylinder (all spanning); and the drag coefficient on non-spanning sections is generally larger than that of the fully non-spanning cylinder. Large mean lift coefficient is observed at cases with small $L$ and $G$, where the span-gap flow is weakened. On the contrary, gap flow tends to be strengthened at large spans, where the lift coefficient is smaller than that on the fullyspanning cylinder.

2) Despite the changes in the sectional forces induced by the geometry difference, the total mean force on the cylinder with intermittent contact can be estimated by a weighted average approach of forces from the spanwise-uniform cylinders. This linear approximation accurately predicts the drag and lift coefficients, within $20 \%$ of difference, and the method is only found to be less accurate when gap flow is encouraged at either large $G$ or large $L$.

3) The sectional difference also leads to approximately four times larger amplitudes in sectional force oscillations as compared to that of spanwise-uniform cylinders, which is due to the variations in gap flow and the turbulence in the flow field. Interestingly, due to the phase difference in sectional forces, the total force on the whole cylinder has small oscillation amplitude.

4) Deflected flow was observed from the non-spanning section to the spanning sections and this has given rise to changes in local force and pressure distributions, especially near the shoulders between spanning and non-spanning sections. 


\section{Acknowledgment}

This work was supported by computing resources provided by the Pawsey Supercomputing Centre with funding from the Australian Government and the Government of Western Australia. H.A. would like to acknowledge the support from the Australian Research Council through DECRA Schemes (DE150100428) and the ECR supporting scheme from the University of Western Australia.

\section{References}

Akoz, M. S., 2012. Investigation of vortical flow characteristics around a partially buried circular cylinder. Ocean Eng., 52, 35-51.

An, H., Cheng, L. \& Zhao, M., 2011. Numerical simulation of a partially buried pipeline in a permeable seabed subject to combined oscillatory flow and steady current. Ocean Eng., 38, $1225-1236$.

Bearman, P. \& Zdravkovich, M., 1978. Flow around a circular cylinder near a plane boundary. $J$. Fluid Mech., 89, 33-47.

Buresti, G. \& Lanciotti, A., 1979. Vortex shedding from smooth and roughened cylinders in crossflow near a plane surface. Aeronautical Quarterly, 30, 305-321.

Cheng, L., An, H., Draper, S. \& White, D., 2016. Effect of wave boundary layer on hydrodynamic forces on small diameter pipelines. Ocean Eng., 125, 26-30.

Cokgor, S. \& Avci, I., 2001. Hydrodynamic forces on partly buried tandem, twin pipelines in current. Ocean Eng., 28, 1349-1360.

Furnes, G. \& Berntsen, J., 2003. On the response of a free span pipeline subjected to ocean currents. Ocean Eng., 30, 1553-1577.

Gao, F.-P., Yan, S.-M., Yang, B. \& Luo, C.-C., 2011. Steady flow-induced instability of a partially embedded pipeline: Pipe-soil interaction mechanism. Ocean Eng., 38, 934-942.

Gao, F., Gu, X. \& Jeng, D. S., 2003. Physical modeling of untrenched submarine pipeline instability. Ocean Eng., 30, 1283-1304.

Germano, M., Piomelli, U., Moin, P. \& Cabot, W. H., 1991. A dynamic subgrid-scale eddy viscosity model. Phys. Fluids, 3, 1760-1765.

Griffiths, T., Shen, W., Xu, M. \& Leggoe, J., 2012. Comparison of Recent Parametric Trenched and Partially Embedded/Spanning Pipelines With DNV-RP-F109 Load Reduction Design Curves. In Proceedings of the 31 st International Conference on Ocean, Offshore and Arctic Engineering, Jul. 1-6, OMAE2012-83282, Rio de Janeiro, Brazil.

Griffiths, T., White, D., Draper, S., Leighton, A. \& Fogliani, A., 2017. Lateral resistance of pipes on rocky seabeds comparison between measurements and models based on synthetic seabeds. In Proceedings of the 36th International Conference on Ocean, Offshore and Arctic Engineering, Jun. 25-30, OMAE2017-61418, Trondheim, Norway.

Jacobsen, V., Bryndum, M. \& Bonde, C., 1989. Fluid loads on pipelines: sheltered or sliding. In Offshore Technology Conference.

Jensen, B., Sumer, B., Jensen, H. \& Fredsoe, J., 1990. Flow around and forces on a pipeline near a scoured bed in steady current. J. Offshore Mech. Arct. Eng., 112, 206-213.

Jeong, J. \& Hussain, F., 1995. On the identification of a vortex. J. Fluid Mech., 285, 69-94.

Kirkil, G. \& Constantinescu, G., 2012. A numerical study of the laminar necklace vortex system and its effect on the wake for a circular cylinder. Phys. Fluids, 24, 073602.

Kozakiewicz, A., Fredsøe, J. \& Sumer, B. M., 1995. Forces on pipelines in oblique attack: steady current and waves. In The Proceedings of the Fifth International Offshore and Polar Engineering Conference, June 11-16, ISOPE-I-95-121, The Hague, The Netherlands.

Leckie, S. H., Draper, S., White, D. J., Cheng, L. \& Fogliani, A., 2015. Lifelong embedment and spanning of a pipeline on a mobile seabed. Coast. Eng., 95, 130-146. 
Leckie, S. H. F., Mohr, H., Draper, S., Mclean, D. L., White, D. J. \& Cheng, L., 2016. Sedimentation-induced burial of subsea pipelines: Observations from field data and laboratory experiments. Coast. Eng., 114, 137-158.

Lei, C., Cheng, L. \& Kavanagh, K., 1999. Re-examination of the effect of a plane boundary on force and vortex shedding of a circular cylinder. J. Wind Eng. Ind. Aerod., 80, 263-286.

Lei, C., Cheng, L. \& Kavanagh, K., 2001. Spanwise length effects on three-dimensional modelling of flow over a circular cylinder. Comput. Methods in Appl. Mech. Eng., 190, 2909-2923.

Nishino, T., Roberts, G. T. \& Zhang, X., 2008. Unsteady RANS and detached-eddy simulations of flow around a circular cylinder in ground effect. J. Fluids Struct., 24, 18-33.

Petruneac, P., 2015. Computational fluid dynamics of turbulence effects on the seabed. Bachelor of Science in Renewable Energy, University of Exeter.

Price, S., Sumner, D., Smith, J., Leong, K. \& Paidoussis, M., 2002. Flow visualization around a circular cylinder near to a plane wall. J. Fluids Struct., 16, 175-191.

Ronold, K. O., 1995. A probabilistic approach to the lengths of free pipeline spans. Appl. Ocean Res., 17, 225-232.

Roshko, A., Steinolfson, A. \& Chattoorgoon, V., 1975. Flow forces on a cylinder near a wall or near another cylinder. DTIC Document.

Sarpkaya, T., 1976. In-Line And Transverse Forces, On Cylinders In Oscillatory Flow At High Reynolds Numbers. In Offshore Technology Conference, May 3-6, OTC 2533, Houston, Texas.

Smagorinsky, J., 1963. General circulation experiments with the primitive equations: I. the basic experiment. Mon. Weather Rev., 91, 99-164.

Sollund, H. A., Vedeld, K. \& Fyrileiv, O., 2015. Modal response of short pipeline spans on partial elastic foundations. Ocean Eng., 105, 217-230.

Sumer, B. M. \& Fredsøe, J., 1997. Hydrodynamics around cylindrical structures. World Scientific, Singapore.

Taniguchi, S. \& Miyakoshi, K., 1990. Fluctuating fluid forces acting on a circular cylinder and interference with a plane wall. Exp. Fluids, 9, 197-204.

Tian, X., Ong, M. C., Yang, J. \& Myrhaug, D., 2014. Large-eddy simulation of the flow normal to a flat plate including corner effects at a high Reynolds number. J. Fluids Struct., 49, 149-169.

Tong, F. \& Cheng, L., 2016. Numerical Study on Steady Flow around a Pipeline Laid on Seabed with Gaps. In The Twelfth ISOPE Pacific/Asia Offshore Mechanics Symposium, Oct. 4-7 ISOPE-P-16-165, Gold Coast, Australia.

Tong, F., Cheng, L. \& Zhao, M., 2015. Numerical simulations of steady flow past two cylinders in staggered arrangements. J. Fluid Mech., 765, 114-149.

Westgate, Z. \& White, D., 2015. Quantifying Spatial Variability of As-Laid Embedment for Subsea Pipeline Design. In Proceedings of the 34th International Conference on Ocean, Offshore and Arctic Engineering, May 31 - Jun. 5, OMAE2015-42292, St. John's, Newfoundland, Canada.

Xu, J., Li, G., Dong, P. \& Shi, J., 2010. Bedform evolution around a submarine pipeline and its effects on wave-induced forces under regular waves. Ocean Eng., 37, 304-313.

Zdravkovich, M., 1985. Forces on a circular cylinder near a plane wall. Appl. Ocean Res., 7, 197201.

Zdravkovich, M. M., 1997. Flow around Circular Cylinders: Volume 1: Fundamentals, Oxford, UK, Oxford University Press.

Zhao, M., Cheng, L. \& Teng, B., 2007. Numerical modeling of flow and hydrodynamic forces around a piggyback pipeline near the seabed. J. Waterw. Port Coast. Ocean Eng., 133, 286295.

Zhao, M., Cheng, L. \& Zhou, T., 2009. Direct numerical simulation of three-dimensional flow past a yawed circular cylinder of infinite length. J. Fluids Struct., 25, 831-847. 